\title{
Participación de las víctimas en la JEP: especial referencia a los informes de las organizaciones de víctimas, étnicas y de derechos humanos*
}

Victims' Participation in the SJP: Special Reference to the Reports of Victims, Ethnic and Human Rights' Organizations

\author{
Lorena Cecilia Vega Dueñas a \\ Pontificia Universidad Javeriana, Colombia \\ vegalorena@javeriana.edu.co \\ ORCID: https://orcid.org/0000-0003-0852-5074
}

DOI: https://doi.org/10.11144/Javeriana.vj69.pvje

Recepción: 07 Octubre 2019

Aceptación: 28 Noviembre 2019

Publicación: 30 Junio 2020

\section{Resumen:}

En la Jurisdicción Especial para la Paz (JEP) de Colombia, las organizaciones de víctimas, étnicas y de derechos humanos pueden presentar un informe en relación a la violación de sus derechos en el marco del conflicto armado (regulados en la Ley 1922 de 2018). Estos informes se consideran una expresión del derecho de participación de las víctimas ante la JEP. Este trabajo, en primer lugar, expone el marco normativo de la JEP en relación a la participación de las víctimas y a su vez plantea la importancia de garantizar este derecho de cara a los retos de esta jurisdicción. En segundo lugar, mediante un abordaje cualitativo, presenta datos recopilados de entrevistas semiestructuradas realizadas a representantes de organizaciones que han presentado informes a la JEP. Estas entrevistas tuvieron como objetivo explorar el derecho de presentar informes a la JEP respecto a tres conceptos: apoyo, visibilidad y participación. Los resultados mostraron que (1) las víctimas reciben apoyo para presentar sus informes, (2) la presentación de los informes aumenta la visibilidad de las víctimas y (3) la presentación de los informes se considera un mecanismo real de participación de las víctimas en la JEP.

Palabras clave: Jurisdicción Especial para la Paz, víctimas, participación, informes.

\section{Abstract:}

In the Colombian Special Jurisdiction for Peace (SJP), victims, ethnic and human rights' organizations can submit a report about the violation of their rights in the framework of the armed conflict (regulated by the Law 1922 of 2018). These reports are considered one expression of the right of victims' participation before the SJP. In this paper, we first present the regulatory framework about the participation of victims in the SJP and the importance of ensuring this right in the face of the challenges of this jurisdiction. Second, we present a qualitative approach of data gathered from semi-structured interviews conducted with representatives of organizations that have submitted reports to the SJP. These interviews aimed to explore the right of submitting reports to the SJP regarding three concepts: support, visibility and participation. Results showed that (1) victims receive support to submit their reports, (2) the submission of the reports increase victims' visibility, and (3) the submission of the reports are considered an actual victims' participation mechanism.

Keywords: Special Jurisdiction for Peace, victims, participation, reports.

\section{Introducción}

La satisfacción de los derechos de las víctimas, en general, y específicamente la participación efectiva, digna y segura de las víctimas de violaciones a los derechos humanos e infracciones del derecho internacional humanitario es un elemento fundamental en un proceso transicional desarrollado en cualquier país. En este sentido se pronunció el Relator especial de las Naciones Unidas sobre verdad, justicia, reparación y no repetición, declarando que: "nada de esto puede suceder a espaldas de las víctimas, sin su valiosa participación. Esta valiosa participación puede revestir diferentes formas (...) Los enjuiciamientos solo serán medidas de

Notas de autor

${ }^{a}$ Autora de correspondencia. Correo electrónico: vegalorena@javeriana.edu.co 
justicia reales si las víctimas y sus familias participan efectivamente en los procesos y reciben la información pertinente necesaria para su participación en los procedimientos judiciales" ${ }^{[1]}$.

Acorde al pronunciamiento del párrafo anterior, la Jurisdicción Especial para la Paz (en adelante JEP), como componente de justicia del Sistema Integral de Verdad, Justicia, Reparación y No Repetición (en adelante SIVJRNR) ${ }^{[2]}$, a través del marco normativo que la rige, se ha encargado de consignar la importancia de la participación de las víctimas en esta jurisdicción y se ha encargado de regular diferentes mecanismos a través de los cuales las víctimas pueden hacer efectivo este derecho. Uno de los instrumentos normativos donde se establecen garantías y derechos que amparan a las víctimas es la Ley 1922 de 2018 por medio de la cual se adoptan las reglas de procedimiento para la Jurisdicción Especial para la Paz (en adelante Ley de procedimiento de la JEP). Específicamente, uno de los derechos reconocidos a las víctimas en esta Ley es el de es presentar informes relativos a las conductas cometidas por causa, con ocasión o en relación directa al conflicto armado ante la JEP, por medio de las organizaciones de víctimas, étnicas y de derechos humanos.

Teniendo en cuenta lo anterior, el presente trabajo tiene como objeto de estudio la participación de las víctimas en la JEP y, específicamente, los informes de las organizaciones de víctimas, étnicas y de derechos humanos como una expresión del derecho a la participación de las víctimas ante esta jurisdicción. Para esto, por un lado, se realiza un estudio de la participación de las víctimas en la JEP, exponiendo las disposiciones normativas esenciales en relación a este derecho y la importancia de su cumplimiento de cara a los retos actuales que enfrenta la jurisdicción. Por otro lado, se indican las nociones principales en relación a los informes y se realiza un abordaje de tipo cualitativo exploratorio de los mismos, presentando datos fruto de entrevistas semiestructuradas a representantes de organizaciones de víctimas, étnicas y de derechos humanos que ya han ejercido el derecho de presentar informes a la JEP.

\section{Participación de las víctimas en la JEP: disposiciones normativas}

La JEP, como componente de justicia del SIVJRNR, será la encargada de investigar, esclarecer, perseguir, juzgar y sancionar las violaciones de derechos humanos e infracciones del derecho internacional humanitario, que tuvieron lugar con anterioridad al 1 de diciembre de 2016 por causa, con ocasión o en relación directa e indirecta con el conflicto armado colombiano, en un plazo de 15 años ${ }^{[3]}$.

La JEP, como justicia transicional, pretende aportar soluciones y herramientas a la sociedad colombiana para enfrentar un legado de graves violaciones a los derechos humanos que tuvieron lugar en el marco del conflicto armado, con el objetivo de alcanzar un futuro más democrático, justo y pacífico ${ }^{[4]}$. De ahí la declaración de que la justicia transicional no solo es un concepto polivante en la medida en que llega a tener múltiples sentidos $^{[5]}$, sino que es una justicia que mira tanto hacia el pasado como hacia el futuro ${ }^{[6]}$.

Desde su negociación hasta su puesta en marcha, la JEP se ha encargado de remarcar su compromiso de actuación teniendo como eje central los derechos de las víctimas ${ }^{[7]}$. La apuesta por la centralidad de las víctimas ${ }^{[8]}$ es coherente con las reformas y medidas que se han adoptado en los últimos años en diferentes países del mundo, con más o menos éxito, las cuales buscan que las víctimas dejen de ser un instrumento más de la maquinaria procesal y cumplan un rol más protagónico en el proceso penal ${ }^{[9]}$. Comprender la centralidad de las víctimas implica entender que los delitos no son solo comportamientos que superan la simple transgresión a un bien jurídico protegido por una norma, sino que también son injusticias cometidas contra seres humanos ${ }^{[10]}$.

Teniendo en cuenta el compromiso de la JEP con los derechos de las víctimas y reconociendo que la participación de las víctimas en el proceso penal se encuentra ligada a la dignidad humana ${ }^{[11]}$, a continuación, presentaremos las disposiciones normativas esenciales en relación a la participación de las víctimas en la JEP, conforme a lo establecido en: el Acuerdo para la terminación del conflicto y la construcción de una paz estable 
y duradera (en adelante Acuerdo de paz), el Acto Legislativo 01 de 2017, por medio del cual se crea un título de disposiciones transitorias de la Constitución para la terminación del conflicto armado y la construcción de una paz estable y duradera y se dictan otras disposiciones (en adelante Acto Legislativo 01 de 2017), la Ley 1957 de 2019 Estatutaria de la Administración de justicia en la Jurisdicción Especial para la Paz (en adelante Ley Estatutaria de la JEP), y en la Ley de Procedimiento de la JEP.

En primer lugar, el Acuerdo de paz dispuso una serie de principios enfocados en las víctimas y sus derechos, que se tendrían en cuenta a lo largo del trabajo para el desarrollo de uno de los seis puntos que lo conforman; específicamente, para el punto 5 sobre las víctimas del conflicto. Uno de los principios establecidos fue la participación de las víctimas ${ }^{[12]}$. En este principio se puso sobre la mesa la necesidad, a través de medios diversos y en momentos diferentes, de que las víctimas de violaciones a los derechos humanos participen en la discusión sobre la satisfacción y restablecimiento de sus derechos. En efecto, como una de las primeras manifestaciones de este principio, el Acuerdo de paz plasmó los mecanismos de participación de las víctimas que se pusieron en marcha durante las negociaciones del punto 5 sobre las víctimas del conflicto ${ }^{[13]}$. Se trataron de foros de víctimas y de testimonios directos de las mismas. Más de 3000 víctimas participaron en los cuatro foros nacionales organizados por la Organización de Naciones Unidas y la Universidad Nacional de Colombia, y sesenta víctimas viajaron a La Habana para entregar sus testimonios y recomendaciones a la Mesa de Conversaciones que se llevaba a cabo en esa ciudad ${ }^{[14]}$.

Igualmente, el Acuerdo de paz ${ }^{[15]}$ contempló el enfoque diferenciado como uno de los elementos básicos del funcionamiento de la JEP. Este enfoque promueve que la JEP haga énfasis en las necesidades de las víctimas mujeres, niñas y niños que han sufrido de una manera desproporcionada y diferenciada los efectos de las graves violaciones de derechos humanos cometidas en el contexto del conflicto y, en consecuencia, haga énfasis en su participación activa y equitativa en la JEP.

El hecho de que la JEP y el SIVJRNR hayan adoptado un enfoque territorial, diferencial y de género ${ }^{[16]}$ pone de manifiesto que, debido a las relaciones desiguales que subordinan a las mujeres y a las identidades de género y a las orientaciones sexuales diversas, se produce discriminación, violencia y aumento en los obstáculos para alcanzar el goce efectivo de los derechos fundamentales ${ }^{[17]}$. Sin duda, este enfoque es un elemento clave para facilitar la participación activa y equitativa ante la JEP de aquellas víctimas que tuvieron un impacto diferenciado y acentuado durante el conflicto armado.

En segundo lugar, el Acto Legislativo 01 de 2017, encargado de crear jurídicamente el SIVJRNR realizó un llamado importante y claro (arts. transitorios 5 y 12), al recordar la importancia de la participación de las víctimas y de los estándares exigidos en esta materia. Este Acto Legislativo realzó la necesidad de que se regulen varios aspectos específicos de la JEP por medio de la ley, entre ellos la participación efectiva de las víctimas con cumplimiento a los principios y garantías procesales según los estándares nacionales e internacionales.

Por una parte, los estándares nacionales a los que hace alusión el Acto Legislativo 01 de 2017 se refieren a los derechos de las víctimas de graves violaciones a los derechos humanos plasmados en la Constitución Política de Colombia de 1991. En este sentido, la Corte Constitucional se ha pronunciado ${ }^{[18]}$, afirmando que

uno de los ejes definitorios de la Constitución de 1991, radica en la obligación del Estado de respetar, garantizar y proteger los derechos humanos de los asociados, lo que incluye el deber de investigar y juzgar de manera seria e imparcial las graves violaciones a los derechos humanos y las infracciones graves al Derecho Internacional Humanitario. El fundamento de este pilar esencial se encuentra en varios de los artículos de la Carta (Preámbulo, arts. 1, 2, 5, 93, 94, 214 y 215-2), en los cuales se señala el compromiso esencial de las autoridades por asegurar a los integrantes de la Nación un conjunto de derechos y libertades básicas y esenciales ${ }^{[19]}$.

Por otra parte, los estándares internacionales a los que hace alusión el Acto legislativo 01 de 2017 se refieren a los derechos de las víctimas de graves violaciones a los derechos humanos plasmados en instrumentos internacionales reconocidos por Colombia, como son: la Declaración Universal de los Derechos Humanos de 1948 (art. 8), el Pacto Internacional de Derechos Civiles y Políticos de 1966 (art. 2), la Convención contra 
la Tortura y otros tratos o penas crueles, inhumanos y degradantes de 1984 (arts. 4, 5 y 6), la Declaración Americana de Derechos y deberes del Hombre de 1948 (arts. 18), la Convención Americana de Derechos Humanos de 1969 (arts. 1, 8 y 25), los Convenios de Ginebra de 1949 (art. 49 Convenio I, art. 50 Convenio 2, art. 129 Convenio 3 y art. 146 Convenio 4) y el Estatuto de Roma de la Corte Penal Internacional de 1998 (art. 17 y 29).

De los anteriores instrumentos internacionales es de especial relevancia la Convención Americana de Derechos Humanos y los pronunciamientos de la Corte Interamericana de Derechos Humanos (en adelante CIDH), ya que este órganos jurisdiccionales, no solo han contribuido de manera representativa al establecimiento de unos estándares normativos que buscan luchar contra la impunidad en casos de violaciones a los derechos humanos ${ }^{[20]}$, sino que han analizado los mecanismos de justicia transicional en contextos de transición de dictadura a democracia y de conflicto armado a paz ${ }^{[21]}$.

En tercer lugar, La Ley Estatutaria de la JEP (art. 18), por un lado, recoge nuevamente el enfoque diferenciado que se había establecido en el Acuerdo de paz ${ }^{[22]}$ como uno de los elementos básicos del funcionamiento de la JEP. Recordemos que este enfoque, como lo expusimos antes, reconoce la importancia de la participación activa y equitativa en la JEP por parte de las mujeres, niñas y niños. Por otro lado, la Ley Estatutaria de la JEP (art. 14), realiza el mismo llamado hecho por el Acto Legislativo 01 de 2017, para que, específicamente, las normas de procedimiento de la JEP contemplen la participación efectiva de las víctimas en los procedimientos de esta jurisdicción, conforme a lo contemplado en los estándares sobre garantías procesales, sustanciales, probatorias, de acceso a un recurso judicial efectivo y demás derechos aplicables. Además de lo anterior, la Ley Estatutaria de la JEP (art. 15) enuncia una serie de derechos de las víctimas, dentro de los cuales destacamos los siguientes, entre otros, por la relación que tienen con la participación de las víctimas: ser reconocidas como víctimas dentro del proceso judicial, aportar pruebas e interponer recursos, e intervenir en las distintas audiencias del proceso.

En cuarto lugar, La Ley de procedimiento de la JEP estableció tres aspectos esenciales en relación a la participación de las víctimas. Primero, la Ley de procedimiento de la JEP (art. 1, b), indicó que las víctimas podrán tener participación en el procedimiento dialógico, es decir, en el procedimiento que se lleva a cabo en casos de reconocimiento de la verdad. Este procedimiento dialógico se aplicará preferentemente sobre el adversarial y se garantizarán los principios y garantías fundamentales en todo caso. Segundo, la Ley de procedimiento de la JEP (art. 3) regula como garantía para la participación de las víctimas el procedimiento para la acreditación de la calidad de víctima. Al respecto, se establece que, después de la recepción de un caso o grupo de casos por parte de la Sala o Sección correspondiente, o después de que la Sala de reconocimiento de verdad realice el contraste de los informes, la víctima que desee participar en las actuaciones deberá presentar prueba de su condición. Finalmente, la Ley de procedimiento de la JEP (art. 27) establece los mecanismos de participación de las víctimas en el procedimiento ante la Sala de reconocimiento de verdad.

La Ley de procedimiento de la JEP en este último precepto (art. 27) enumera una serie de derechos de las víctimas, dentro de los cuales destacamos los siguientes, entre otros, por la relación que tienen con la participación de las víctimas: ser oídas en los supuestos de priorización y selección de casos; aportar pruebas; presentar observaciones finales escritas a la resolución de conclusiones; presentar observaciones en relación con los proyectos restaurativos; y, finalmente, el derecho y mecanismo de participación de las víctimas ante la Sala de reconocimiento de verdad, en el cual centra su atención este trabajo, la presentación de informes por medio de las organizaciones de víctimas, indígenas, negras, afrocolombianas, raizales, palenqueras y Rrom y de derechos humanos. 


\section{Participación de las víctimas en la JEP: elemento esencial en el proceso transicional}

En la actualidad, se parte de la concepción de que todo proceso transicional debe permitir la participación de las víctimas, o de lo contrario se dificultará alcanzar una reconciliación social y un respaldo nacional e internacional. En otras palabras, la participación de las víctimas es un elemento esencial, una condición sine qua non de un proceso transicional que pretenda ser eficaz, sólido y acreditado ${ }^{[23]}$.

En este sentido, consideramos que la participación de las víctimas en la JEP es un componente fundamental para afrontar muchos de los retos más importantes de esta jurisdicción. Entre ellos: dignificar, visibilizar y reconocer a las víctimas de las graves violaciones a los derechos humanos; validar a las víctimas de graves violaciones a los derechos humanos; aumentar la legitimidad de la jurisdicción; acrecentar la transparencia de la jurisdicción; y desarrollar de manera idónea el componente restaurativo de la jurisdicción.

Frente al reto de dignificar, visibilizar y reconocer a las víctimas consideramos que su participación es esencial ${ }^{[24]}$. Su participación en los procedimientos de la JEP restaura su dignidad, porque son ellas mismas las que aportan al proceso testimonios o evidencias para esclarecer la verdad ${ }^{[25]}$. De igual manera, la participación de las víctimas en los procedimientos de la JEP les brinda visibilidad porque permite que sean ellas las protagonistas cuando dan a conocer su historia y su sufrimiento, además de reconocerse y aumentar su sentido del poder como parte del proceso.

En segundo lugar, la participación de las víctimas es un elemento primordial para que la JEP haga frente al reto de validarlas ${ }^{[26]}$. Es decir, la participación de las víctimas en la JEP permite validar el relato y el coraje de las personas que deciden contarle al país su caso y su drama. La JEP debe validar a las víctimas con el fin de que se sientan lo suficientemente seguras para expresar su voz en los asuntos que les afectan, es decir, con el fin de que se apropien de su propia voz ${ }^{[27]}$. Al respecto, se ha afirmado que es imprescindible darles la palabra a las víctimas si no queremos fracasar en el intento de paz ${ }^{[28]}$.

En tercer lugar, la participación de las víctimas es un elemento primordial para que la JEP haga frente al reto de aumentar su legitimidad ${ }^{[29]}$. Así, el hecho de que participen aquellas personas que han sufrido afectaciones de todo orden en los procedimientos de la JEP indica que las víctimas reconocen esta jurisdicción y su marco legal ${ }^{[30]}$. Por el contrario, la ausencia de las víctimas en procesos transicionales le quita legitimidad al proceso en la medida en que las víctimas no se apropian del mismo. Sin duda, la JEP estará vigente mientras las víctimas estén en el centro de su implementación normativa y de la puesta en marcha de los mecanismos, instancias y medidas creadas ${ }^{[31]}$.

En la consecución de la transparencia de la jurisdicción, entendida como aquella cualidad que hace que esta sea clara, evidente y se comprenda sin duda ni ambigüedad, influyen varios elementos internos y externos, teóricos y prácticos; y uno de estos elementos lo constituye la participación de las víctimas en los procedimientos de la JEP. El hecho de que las víctimas participen presenta la ventaja de que son ellas mismas las que acompañan y realizan el control de las diferentes etapas del proceso, con el fin de evitar la violación, tanto de sus derechos, como de los principios y garantías fundamentales ${ }^{[32]}$. Cuando las víctimas participan en contextos de justicia transicional aumenta la probabilidad de que sus necesidades sean atendidas y de que el proceso se integre mejor en otros objetivos de la justicia transicional como la verdad y la reparación ${ }^{[33]}$.

Finalmente, en quinto lugar, la participación de las víctimas es un elemento primordial para que la JEP haga frente al reto de desarrollar de manera idónea el componente restaurativo ${ }^{[34]}$. En este punto debemos partir diciendo que, tanto el Acuerdo de paz ${ }^{[35]}$, el Acto Legislativo 01 de 2017 (art. transitorio 1), la Ley Estatutaria de la JEP (art. 4 y 13) como la Ley de procedimiento de la JEP (art. 1) se encargaron de establecer que, con el fin de garantizar los presupuestos necesarios para la reconciliación y para el establecimiento de una paz estable y duradera, uno de los principios orientadores de la JEP sería la aplicación de una justicia restaurativa. 
Dicho esto, la participación de las víctimas en la JEP permite desarrollar de manera idónea el componente restaurativo porque, por una parte, la participación encarna uno de los valores de la justicia restaurativa de acuerdo a una de las definiciones más difundida y aceptada internacionalmente, la cual afirma que la justicia restaurativa es un conjunto de procesos y resultados que giran en torno a cuatro valores: encuentro, reparación, reintegración y participación ${ }^{[36]}$. El primero es el encuentro entre las partes, víctima, victimario y su comunidad; el segundo es la reparación del daño ocasionado a la víctima; el tercero es la reintegración a la sociedad, tanto de la víctima como del infractor; y el cuarto es la participación de las partes, víctima e infractor y, si es posible, la comunidad en la resolución del conflicto. Por otra parte, la participación hace parte de una dinámica procesal cooperativa propia del enfoque restaurativo en procesos transicionales. En esta línea, la justicia restaurativa en contextos de justicia transicional ha sido entendida ${ }^{[37]}$ como una aproximación pacífica y colaborativa para resolver el conflicto, en busca del establecimiento de responsabilidades, de sanación y de justicia $^{[38]}$.

En todo caso, bien se ha reconocido que hacer justicia restaurativa real en un contexto transicional es muy difícil. La JEP tiene el gran desafío de demostrarle al mundo que el componente restaurativo sí es adecuado para sancionar las graves violaciones a los derechos humanos ${ }^{[39]}$. Sea como sea, un primer paso importante para encarar este desafío es establecer y garantizar la participación efectiva de las víctimas en la JEP.

\section{Informes de las organizaciones de víctimas, étnicas y de derechos humanos: concepto, rasgos definitorios y requisitos}

En las siguientes páginas centraremos nuestra atención en uno de los derechos y mecanismos de participación de las víctimas ante la JEP, regulado en la Ley de Procedimiento de la JEP (art. 27), el cual consiste en la presentación de informes a la Sala de reconocimiento de verdad por medio de las organizaciones ${ }^{[40]}$ de víctimas, indígenas, negras, afrocolombianas, raizales, palenqueras y Rrom y de derechos humanos ${ }^{[41]}$.

Estos informes han sido definidos como un mecanismo de acceso a la justicia en la JEP y una primera expresión del derecho a la participación de las víctimas ante la JEP. A través de ellos, por un lado, se dan a conocer los hechos que, a juicio de las organizaciones, constituyeron graves violaciones a los derechos humanos y, por otro lado, se les reconoce la capacidad a las víctimas para que participen activamente en los procesos judiciales $^{[42]}$. Así, de acuerdo al Auto 222 de 8 de octubre de 2019 de la JEP, a esa fecha se habían presentado 214 informes ante la Sala de reconocimiento de verdad, de los cuales 101 (47\%) fueron suscritos por organizaciones de víctimas, étnicas y de derechos humanos.

Los informes son una pieza fundamental para el trabajo de la Sala de reconocimiento de verdad porque estos contribuyen a que la Sala, entre otras cosas: primero, contraste la información recibida de la Fiscalía General de la Nación, la Justicia Penal Militar, la Jurisdicción Especial Indígena, la Procuraduría General de la Nación, la Contraloría General de la República, la Administración de la Rama Judicial y de cualquier jurisdicción que opere en Colombia; segundo, realice análisis de contexto y de patrones de las violaciones a los derechos humanos cometidas durante el conflicto armado interno; y tercero, identifique a los presuntos responsables de dichas conductas criminales, así como a las víctimas para efectos de su participación activa ante la JEP ${ }^{[43]}$.

Los informes se podían presentar, en principio, entre el 15 de marzo de 2018 y el 15 de marzo de 2020; no obstante, la JEP, por medio del Auto 222 de 8 de octubre de 2019, prorrogó este plazo hasta el 15 de marzo de 2021. La presentación de estos informes, de manera general, se puede hacer por medio físico o preferiblemente por medios digitales (correo electrónico, USB, CD, etc.), pero también hay dos particularidades que quisiéramos destacar ${ }^{[44]}$. Por un lado, con la intención de dar mayor visibilidad a las víctimas y de que la presentación de los informes sea un hecho restaurador en sí mismo, la entrega y recepción de los mismos se podrá hacer por medio de actos públicos y simbólicos en la JEP frente a los magistrados y 
magistradas de esta jurisdicción ${ }^{[45]}$. Por otro lado, en atención al impacto diferenciado del conflicto armado sobre determinadas personas y en determinados territorios, se contemplan consideraciones especiales en relación a los informes sobre violencia basada en género, violencia en contra de niños, niñas y adolescentes y en relación a los informes presentados por pueblos étnicos. En este último caso, por ejemplo, los informes podrán tener un carácter mixto (escrito y oral), con soportes de grabaciones de voz u otros medios, y podrán ser recibidos en sus territorios ${ }^{[46]}$. Para ello se contará con el apoyo de los enlaces territoriales ${ }^{[47]}$ y del equipo de la dependencia para la participación de las víctimas de la Secretaría Ejecutiva de la JEP.

Los informes podrán activar la competencia de la JEP para la investigación de los hechos delictivos allí consignados. Una vez recibidos por parte de la Sala de reconocimiento de verdad se procederá a contrastarlos con otra información recolectada por diferentes medios (otros informes, versiones voluntarias, acervo probatorio ${ }^{[48]}$. En otras palabras, la JEP no podrá declarar responsabilidades penales únicamente a partir del contenido de los informes que presenten las organizaciones ${ }^{[49]}$. Además, es fundamental resaltar que la presentación de informes a la Sala de reconocimiento de verdad no implica la pérdida automática e inmediata de la competencia que tiene la Fiscalía General de la Nación o demás autoridades ordinarias para investigar los hechos allí relatados. Solamente cuando la Sala de reconocimiento de verdad anuncia públicamente la presentación ante el Tribunal para la Paz de la resolución de conclusiones respectiva, la Fiscalía o el órgano investigador correspondiente pierde la competencia para continuar investigando los hechos o conductas competencia de la JEP ${ }^{[50]}$.

Los informes deberán cumplir unos requisitos básicos y podrán cumplir otros requisitos adicionales $^{[51]}$. Por una parte, los requisitos básicos que deben cumplir los informes son los siguientes: identificar a la organización; realizar una relación descriptiva de los hechos ocurridos con ocasión del conflicto armado colombiano de los cuales la organización posea información concreta; y señalar si la información presentada fue puesta en conocimiento de alguna otra autoridad. Por otro lado, aparte de los requisitos básicos anteriores, los informes también podrán, de manera optativa, cumplir los siguientes requisitos: agrupar los hechos semejantes en una misma categoría; organizar la información según los hechos más representativos; describir los hechos de manera completa (contexto, lugar, fecha, modus operandi); identificar presuntos autores y víctimas de los hechos relatados; describir los daños e impactos generados; describir la metodología utilizada para la agrupación de la información; aportar información para identificar procesos adelantados ante otra autoridad pública; solicitar el anonimato de quienes remiten el informe; relacionar si las víctimas han elevado solicitud de reparación ante otra autoridad competente; presentar valoraciones jurídicas de los hechos de manera separada de la descripción de los hechos; y manifestar el objetivo perseguido con la presentación del informe.

\section{Informes de las organizaciones de víctimas, étnicas y de derechos humanos: principales hallazgos de las entrevistas}

En relación a los informes, realizamos un abordaje de tipo cualitativo exploratorio. De esta manera, presentamos datos de entrevistas semiestructuradas que, sin la intención de considerarse generalizables, sí se podrían tomar como una primera exploración o un primer panorama de este mecanismo de participación de las víctimas en la JEP. Estas entrevistas semiestructuradas tuvieron como guía tres aspectos sobre los cuales indagamos. La selección de estos tres aspectos se hizo teniendo en cuenta que la justicia restaurativa es un principio básico para la formulación e implementación de la JEP ${ }^{[52]}$ y que el primer objetivo de este tipo de justicia, de acuerdo con las Naciones Unidas, es "apoyar a las víctimas, darles una voz, motivarlas a expresar sus necesidades, permitirles participar en el proceso de resolución y ofrecerles ayuda" ${ }^{[53]}$. Es decir, acorde con las Naciones Unidas ${ }^{[54]}$, un modelo restaurativo es aquel que apoya un proceso en donde las visiones 
e intereses de las víctimas cuentan, en donde ellas pueden participar, en donde ellas son tratadas de manera justa y respetuosa, en donde ellas puedan recibir una restauración o indemnización, y en donde ellas tengan voz en la determinación del resultado del proceso.

En consecuencia, el primer aspecto sobre el cual indagamos fue apoyo. Apoyo, entendido en la acepción de "protección, auxilio o favor", de acuerdo con la Real Academia Española. Es decir, para este trabajo, y así fue manifestado a los entrevistados, el apoyo hace referencia a la ayuda o colaboración que han podido recibir las víctimas para la elaboración y presentación de los informes a la JEP. El segundo aspecto sobre el cual indagamos fue la voz o visibilidad. Visibilidad, entendida en la acepción de la Real Academia Española como "cualidad de visible". Es decir, para este trabajo se le manifestó a los entrevistados que la visibilidad hace referencia a la notoriedad e importancia que han podido adquirir las víctimas debido a la elaboración y presentación de los informes a laJEP. Finalmente, el tercer aspecto sobre el cual indagamos fue la participación, entendida como la "acción y efecto de participar", de acuerdo con la Real Academia Española. Es decir, que a los entrevistados se les explicó que la participación hace referencia a la cooperación o intervención que han podido tener las víctimas en la elaboración y presentación de los informes a la JEP y como estos han sido reconocidos como un mecanismo de participación ante esta jurisdicción.

Las entrevistas semiestructuradas fueron realizadas a siete representantes de organizaciones de víctimas, étnicas y de derechos humanos ${ }^{[5]}$, las cuales han presentado al menos un informe a la JEP. Estas organizaciones son las siguientes: Movimiento Nacional de Víctimas de Crímenes del Estado (en adelante Movice), Asociación para la Promoción Social Alternativa Minga (en adelante Minga), Coalición contra la vinculación de niños, niñas y jóvenes al conflicto armado en Colombia (en adelante Coalico), Madres de Falsos Positivos en Soacha y Bogotá (en adelante Mafapo), Fundación Visión Desarrollo Social (en adelante Funvides), Fondo de Solidaridad con los Jueces colombianos (en adelante Fasol) y Colombia Diversa.

Para conocer cómo seleccionamos a las personas entrevistadas debemos tener en cuenta los siguientes datos. La información sobre las organizaciones de víctimas, étnicas y de derechos humanos que ya presentaron informe a la JEP no es pública. Es público el número de informes presentados que, como indicamos anteriormente, de acuerdo al Auto 222 de 8 de octubre de 2019 de la JEP, a esa fecha se habían presentado 214 informes ante la Sala de reconocimiento de verdad, de los cuales 101 (47\%) fueron suscritos por organizaciones de víctimas, étnicas y de derechos humanos. Sin embargo, como también lo indicamos anteriormente, algunas organizaciones deciden hacer uso de la posibilidad de realizar un acto público y simbólico el día que entregan el informe a la JEP. A todas las organizaciones de las cuales existía alguna noticia -hasta julio de 2019, momento en el que se realizó esta investigación-, ya sea emitida por parte de la propia JEP o por parte de los medios de comunicación, sobre la presentación del informe a través de acto público y simbólico, se les envió una carta de invitación vía email para participar como entrevistados. De las veintitrés organizaciones invitadas, siete contestaron al llamado, seleccionaron un representante y tuvieron la disponibilidad para concedernos la entrevista ${ }^{[56]}$. Todas las personas entrevistadas fueron, por lo tanto, seleccionadas intencionalmente. La entrevista, la cual tenía una duración de 30 a 45 minutos, comenzaba con la explicación de los rasgos esenciales de la investigación, continuaba con la lectura y firma del consentimiento informado y terminaba con la realización de las preguntas sobre los tres aspectos guía y sobre aquellos elementos que fueran llamando la atención del entrevistador a medida que se desarrollaba la entrevista. Todas las entrevistas fueron grabadas en audio con la autorización de los entrevistados y posteriormente transcritas y analizadas con la ayuda del software NVivo. De esta manera, una vez explicados los detalles metodológicos de este trabajo, a continuación, presentamos los principales resultados de las entrevistas semiestructuradas. 


\section{Principales hallazgos de entrevistas: apoyo}

En primer lugar, todos los entrevistados coinciden en que las víctimas sí han recibido apoyo para la realización y presentación de los informes (7/7 entrevistados). No obstante, este apoyo ha venido de la mano de cada organización que se ha puesto al frente de la realización y presentación de los informes ( $7 / 7$ entrevistados), en algunos casos por parte de la propia JEP ( $4 / 7$ entrevistados) o en un caso ha venido por parte de una Organización no gubernamental (1/7 entrevistados). En todo caso, aunque sí se reconoce un apoyo, se hace notar que este es insuficiente y que, principalmente, debería ser reforzado por instituciones del Estado (5/7 entrevistados); por ejemplo: Defensoría del Pueblo, Procuraduría General de la Nación y Personerías. Este apoyo prestado por las instituciones del Estado, de acuerdo con la opinión de las personas entrevistadas, podría ir dirigido a dos fines principales. Por un lado, de acuerdo con los representantes entrevistados de Colombia Diversa, Fasol, Coalico y Minga, se podría apoyar otorgando información clara y pertinente sobre los informes como mecanismo de participación de las víctimas ante la JEP y/o realizando actividades pedagógicas sobre sus requisitos, elaboración y presentación, especialmente en municipios pequeños. Por otro lado, de acuerdo con el representante entrevistado de Funvides, se podría apoyar convocando a las principales organizaciones de víctimas, étnicas y de derechos humanos para respaldar y agilizar la elaboración y presentación de los informes a la JEP, con el fin de que estos sean lo más sólidos y útiles posibles para la jurisdicción.

En relación a lo anterior, compartimos el siguiente fragmento de una entrevista:

Hemos recibido asesoría u orientación por parte de la JEP; hemos hablado con varios directivos y con la Secretaría ejecutiva y sí hay orientación (...) Por otra parte, el Ministerio Público ha tenido un rol importante en todo el tema de justicia transicional en Colombia y uno esperaría que la Procuraduría dijera: vamos a realizar informes de la mano con las organizaciones de víctimas. Sin embargo, esto no ha sido posible. No ha habido una convocatoria de la Procuraduría o de la Personería en este sentido. (Funvides)

De acuerdo con el representante entrevistado de Fasol, el hecho de que las víctimas no reciban mayor apoyo de las instituciones del Estado como Fiscalía General de la Nación, Defensoría del Pueblo o Procuraduría General de la Nación ${ }^{[57]}$ se explica por varias razones, entre ellas: el nivel de desconocimiento de la JEP por parte de los funcionarios de estas entidades es alto; que no hay un interés real y generalizado por esta jurisdicción; que no hay recursos humanos suficientes, lo que hace que la JEP sea vista como una carga laboral más; y que hay un problema generacional en los funcionarios de estas entidades. Es decir,

hay un choque entre los que son nuevos, que vienen con una idea de la justicia transicional, y los que son un poco más antiguos, que creen por ejemplo que la Ley 600 es la ley que debe funcionar para estos casos, sin entender toda la estructura sociojurídica que debe tener un sistema de justicia transicional. (Fasol)

Al guardar relación con las razones que pueden determinar el apoyo o no a la realización y presentación de informes, los representantes entrevistados de Coalico, Fasol y Funvides identifican como un punto fundamental la falta de recursos económicos para la realización de los mismos, y reconocen de la importancia del patrocinio por parte de la cooperación internacional para que muchos informes hayan podido entregarse exitosamente. Concretamente, se hizo alusión a la cooperación holandesa, alemana y a la cooperación por parte de la Misión de Apoyo al Proceso de Paz en Colombia de la Organización de los Estados Americanos (MAPP-OEA).

Alrededor de este primer aspecto -apoyo-, las entrevistas realizadas nos permitieron identificar los siguientes dos elementos: falta de pedagogía para los informes y superposición de información relacionada con los procesos de justicia transicional en el país.

Por una parte, las personas entrevistadas ponen de manifiesto la falta de pedagogía para los informes, especialmente al nivel de complejidad de los requisitos exigidos para los mismos ${ }^{[58]}$. En efecto, se afirmó que: 
la metodología de acompañamiento de esos informes es casi nula. A uno le entregan la cartilla y le dicen cómo hacerlo, pero digamos que una revisión periódica o una versión preliminar no hay (...) Ahí hay un vacío grandísimo, de la información a la presentación del informe hay un abismo grandísimo. (Fasol)

Inicialmente, el representante entrevistado de Colombia Diversa expresa que muchas víctimas no han recibido información ni capacitación en relación a su derecho a presentar informes y que las actividades divulgativas o de capacitación al respecto se han desarrollado principalmente en Bogotá o en las grandes ciudades. En este punto, desde nuestro punto de vista, se hace evidente el hecho de que la JEP tenga su sede principal en Bogotá y que solo estuviera intentando hacer presencia en territorio a través de los enlaces territoriales. Esta situación ha cambiado recientemente -segundo semestre 2019- debido a que la jurisdicción abrió tres sedes fuera de la capital (Corozal, Sucre; Cúcuta, Norte de Santander y Pasto, Nariño $)^{[59]}$. Posteriormente, el representante entrevistado de Minga expresa que los informes que está esperando la JEP son difíciles de elaborar y son especialmente técnicos. Además, se reitera la idea de que, precisamente por la situación anterior, las víctimas que no pertenecen a una organización consolidada que pueda apoyar el proceso de realización del informe, difícilmente podrán presentar uno.

En relación a lo anterior, compartimos el siguiente fragmento de una entrevista:

No existe una pedagogía ni siquiera para llegar a una Audiencia, pues mucho menos hay una pedagogía que permita conocer el derecho a informes; que son complejos, que, si una víctima no se agrupa a una organización o a una Junta, o se organiza de alguna forma, no va a tener la posibilidad de presentarlos. (Minga)

El hecho de que, al parecer, solo las víctimas que pertenecen a organizaciones consolidadas puedan presentar informes a la JEP puede cuestionar sobre si existe o no una violación al acceso a la justicia como principio básico de un Estado de Derecho. Sin embargo, en las entrevistas realizadas se identifica esta opción como la mejor, pues la JEP no tendría capacidad para relacionarse con las víctimas individuales, aisladas o campesinas. En otras palabras:

el hecho de que el interlocutor de la JEP sean las organizaciones es lo más viable (...) Además, es completamente entendible que el informe deba tener un sentido, que no sea sencillo. No se trata de presentar un informe por presentarlo, pues para decir que hay víctimas se van al registro único de víctimas; la finalidad del informe debe ser impactar realmente al sistema. (Funvides)

Desde nuestro punto de vista, la JEP sí aspira a que las organizaciones de víctimas, étnicas y de derechos humanos sean sus interlocutores, aspira a que de esta relación las organizaciones salgan fortalecidas y aspira a que, a través de la información que le presenta estas organizaciones, esta jurisdicción pueda comprender el proceso en clave territorial. En este punto merece la pena preguntarse si quizás lo indicado es ser consciente de que una jurisdicción como la JEP tendrá resultados de impacto, no de números.

Por otra parte, en las entrevistas realizadas se pone de manifiesto la superposición de información relacionada con los procesos de justicia transicional en el país. Así, de acuerdo con el representante entrevistado de Colombia Diversa, en los últimos años han existido múltiples talleres para las víctimas de graves violaciones a los derechos humanos acerca de varios mecanismos de justicia transicional, de la mano de diferentes organizaciones o instituciones, pero que la comprensión real y efectiva de esta información por parte de la comunidad es baja. En otras palabras, según el representante de Minga, muchas víctimas no ven la diferencia entre acudir a la Unidad de Víctimas, a Justicia y Paz o a la JEP, en gran parte por la sobresaturación o exceso de información en relación a los procesos de justicia transicional, pero también por la pérdida de confianza en el Estado. En relación a lo anterior, compartimos el siguiente fragmento de una entrevista:

Hay una sobresaturación de información, sea de talleres o del Estado tratando de acercarse a las víctimas a darle información; pero a pesar de eso, hay una muy baja comprensión de esa información por parte de las víctimas (...) Yo creo que las víctimas no se sienten tan cercanas a la JEP precisamente por esa dualidad entre la mucha información, pero también la mucha incomprensión de esa información. (Colombia Diversa) 


\section{Principales hallazgos de entrevistas: visibilidad}

En segundo lugar, todos los entrevistados coinciden en que la presentación de los informes sí les ha dado visibilidad a las víctimas ( $7 / 7$ entrevistados), especialmente ante la sociedad colombiana ( $4 / 7$ entrevistados), los medios de comunicación ( $2 / 7$ entrevistados) y la comunidad internacional (3/7 entrevistados). con respecto a lo anterior, compartimos los siguientes fragmentos de las entrevistas:

De alguna manera sí hemos sido más visibles porque cuando presentamos este informe con un acto público se conoció a través
de los medios. Igual saben que nosotras aún estamos ahí, dando esa batalla. Entonces sí nos ha dado más visibilidad (Mafapo).
No sabemos cuándo se volverá a presentar esta oportunidad de que la JEP consiga verdad, justicia, reparación y garantías de
no repetición, para que a ningún ciudadano colombiano le ocurra lo que le ocurrió a nuestros seres queridos, que tengan que
cargar el dolor que llevamos por muchos años y que seguimos llevando (...); entonces creo que la presentación de informes
por parte de las organizaciones de víctimas sobre lo ocurrido en el conflicto nos va dar visibilidad ante la sociedad colombiana
y ante la comunidad internacional. (Movice).

Sin embargo, aunque es unánime la idea de que las víctimas ganan visibilidad a través de la presentación de los informes, se deja constancia de dos particularidades: la visibilidad no es para todas las víctimas y la visibilidad es efímera. La primera de ella se refiere a que, de acuerdo con el representante entrevistado de Minga, la visibilidad no es para todas sino solo para aquellas víctimas que pertenecen a una organización consolidada. No obstante, el representante de Colombia Diversa, reconoce que esta visibilidad también es importante porque, aunque no se visibilicen individuos, se visibilizan preguntas (¿Existió? ¿Por qué? ¿Cómo? ¿Cuándo?), violencias y memorias. En todo caso, se afirma que el grado de visibilidad que sienten las víctimas con la presentación de los informes está relacionado con la participación que hayan tenido en el mismo. Sin duda, es fundamental que las víctimas sean el eje central de sus propias historias. En otras palabras:

la conexión que puedan sentir las víctimas con esa presentación de informes es completamente proporcional a la participación en el mismo. Y esto lo digo porque nosotras el primer compromiso que tuvimos con las víctimas fue ser súper transparentes con lo que íbamos a presentar, mostrarles como habían quedado sus historias, como iban a ser incluidas en los informes, y que ellos en últimas tuvieran el poder de decidir cómo y qué quieren contar. (Colombia Diversa)

La segunda particularidad se refiere a que la visibilidad que se haya podido obtener para las víctimas es momentánea. En otras palabras:

la respuesta a la pregunta de si las víctimas ganan visibilidad con la presentación de informes es sí y no, lamentablemente. De parte de la comunidad internacional hay más receptividad, de los países amigos, del Consejo de seguridad; hay más reconocimiento y espacios muy relevantes. No obstante, en la cotidianidad del día a día de Colombia, en las noticias, pasa desapercibido. (Coalico).

Alrededor de este segundo aspecto -visibilidad-, las entrevistas realizadas nos permitieron identificar los siguientes dos elementos: el plazo de los informes próximo a vencerse y la limitación en el acceso a la información para las organizaciones.

Por un lado, en las entrevistas realizadas se revela que el plazo dado por la JEP para la presentación de informes ha sido muy corto, ya que, al momento de realizar las entrevistas la fecha de vencimiento era marzo de 2020. No obstante, de acuerdo al Auto 222 de 8 de octubre de 2019 de la JEP, el plazo fue ampliado hasta marzo de 2021. En efecto, se afirmó en relación a la JEP: "ellos mismos pusieron el tiempo y ellos mismos se están castigando" (Fasol). Desde nuestro punto de vista, un plazo corto para la presentación de los informes puede tener dos consecuencias esenciales. La primera es que muchos de los informes que se presenten no hayan tenido suficiente tiempo para su realización y se queden en lo narrativo, es decir, no brinden herramientas suficientes a la jurisdicción para abrir un caso. La segunda consecuencia es que muchos informes no alcancen a presentarse y con ello muchas de las graves violaciones a los derechos humanos, contadas por sus propias víctimas, se queden sin registro. Lo anterior debe llamar nuestra atención si tenemos en cuenta que es prioritario recoger la información sobre los hechos desde el cristal de las víctimas, desde 
su análisis de contexto, desde sus relaciones o conexiones de los hechos y desde sus criterios de gravedad e impacto.

En relación a lo anterior, compartimos el siguiente fragmento de una entrevista: "Una gran dificultad es el término, que para presentar informes se vence en marzo de 2020, es decir, prácticamente estamos ad portas del cierre y se quedarán bastantes informes sin presentar" (Minga).

Por otro lado, en las entrevistas realizadas se revela que el acceso a la información es limitado. Así, las organizaciones pueden tener toda la intención de plasmar, organizar y clasificar la información recopilada en relación a las graves violaciones a los derechos humanos, pero no siempre tienen acceso a la información por motivos de seguridad, de reserva sumarial, de reserva procesal, entre otras. Con respecto a esto, el representante de Fasol nos indica:

Una cosa que sí nos sucedió es la siguiente. Este país es muy centralizado. Nosotros teníamos muchos casos en los llanos orientales que queríamos documentar y fue imposible conseguir la información. Ni la defensoría, ni las alcaldías, ni las gobernaciones sabían, es como si se hubiera borrado esa información. Nosotros le solicitamos información a la Fiscalía sobre esos ilícitos/tipos penales y no la daban tampoco. Decían: en nuestra base de datos no reporta esa información, esa es la respuesta que dan. Yo creo que hay unos vacíos. Ahí yo creo que se reafirma ese sentimiento que desafortunadamente hay de escepticismo y de poca fe a esta justicia (Fasol).

\section{Principales hallazgos de entrevistas: participación}

Todos los entrevistados coinciden en que los informes sí han sido reconocidos como un mecanismo de participación para las víctimas ( $7 / 7$ entrevistados/as). No obstante, aunque es unánime la idea anterior, se deja constancia de que el derecho a la presentación de informes es un punto de partida acertado y destacable, pero no basta, la participación de las víctimas en la JEP es más compleja y debe darse en todas las fases del proceso (3/7 entrevistados/as).

$\mathrm{Al}$ respecto, nos indica un representante: "Sí claro, de todas maneras, en la justicia ordinaria las víctimas no podían decir nada, casi ni respirar. Si las escuchaban hablando las sacaban de las audiencias. Por lo menos en la JEP se tienen más maneras de participación” (MAFAPO).

De acuerdo con la opinión de las personas entrevistadas, los informes son reconocidos como un mecanismo de participación para las víctimas por dos razones principales. En primer lugar, porque, en opinión del representante de Colombia Diversa, los informes, independientemente de que constituyan la herramienta para abrir un caso o no, significan un acercamiento entre las víctimas y el Estado, específicamente entre las víctimas y la JEP. En segundo lugar, porque, en opinión del representante entrevistado de Coalico, los informes son concebidos por la JEP como la posibilidad de que las víctimas participen en la selección de los casos.

Con respecto al aspecto de la participación, las entrevistas realizadas nos permitieron identificar los siguientes dos elementos: la participación de las víctimas en la JEP encierra varios componentes y la carga adicional generada para las víctimas debido a los informes.

Por una parte, las entrevistas realizadas dejan constancia de que la participación de las víctimas, para que sea efectiva, implica la unión de varios componentes, entre ellos la acreditación de calidad de víctima o la representación judicial. En relación a lo anterior, compartimos el siguiente fragmento de una entrevista: "Yo pienso que la presentación de informes es alzar la mano, pero la participación efectiva es la presentación del informe con su solicitud de acreditación individual; con la presentación del informe no se agota la participación" (Funvides).

Por otra parte, las entrevistas realizadas dejan constancia de que la realización y presentación de los informes es un mecanismo de participación para las víctimas, pero que dependiendo del cómo, quién y dónde del mismo, genera unas cargas adicionales para ellas mismas. Al respecto uno de los representantes opina: 
Entonces, claro que es un mecanismo de participación, pero creo que es un mecanismo de participación que dependiendo de quién y cómo se decida participar, genera unas cargas. Unas cargas sobre las víctimas que yo no sé si sean cargas razonables. Por ejemplo, yo sí creo que así la JEP diga que los informes son sencillos y no hay mucho trabajo que hacer, yo creo que esos informes requieren de un trabajo técnico que la gran mayoría de víctimas no está en la disposición de hacer, y eso genera desigualdad entre las víctimas (Colombia Diversa).

\section{Conclusiones}

El marco normativo de la JEP ha reflejado la importancia y la preocupación porque este proceso transicional tenga en cuenta y proteja los derechos de las víctimas de violaciones a los derechos humanos e infracciones al derecho internacional humanitario en el marco del conflicto armado colombiano. Este cometido comenzó desde el propio Acuerdo de paz donde se estableció la participación de las víctimas como un principio orientador del funcionamiento de la JEP y ha continuado desarrollándose a medida que se ha implementado el Acuerdo de paz a través de diferentes leyes.

En este sentido, hasta el momento, la Ley de procedimiento de la JEP ha establecido los elementos más específicos en relación a la participación de víctimas y regulado el procedimiento dialógico, la acreditación de calidad de víctima y diferentes mecanismos para su participación en el procedimiento ante la Sala de reconocimiento de verdad, entre ellos, el derecho a presentar informes por parte de las organizaciones de víctimas, étnicas y de derechos humanos.

De igual manera, basados en la opinión de diferentes expertos en justicia transicional, especialmente en la opinión de los líderes de los diferentes mecanismos que componen el SIVJRNR, pudimos observar la importancia de la participación de las víctimas en la JEP al afrontar y superar varios retos actuales de esta jurisdicción. Nos referimos, por un lado, a los retos relacionados con las propias víctimas, como conseguir su dignificación, visibilización, reconocimiento y validación y, por otro lado, nos referimos a los retos relacionados con la propia jurisdicción, como fortalecer su legitimidad, transparencia y aplicación del enfoque restaurativo.

En relación a los informes de las organizaciones de víctimas, étnicas y de derechos humanos, pudimos hallar varios elementos y percepciones interesantes y comunes en relación a este mecanismo de participación de las víctimas ante la JEP a través de las entrevistas realizadas.

En primer lugar, de acuerdo a las respuestas de las personas entrevistadas, podemos afirmar que las víctimas sí han recibido apoyo para la realización y presentación de los informes, fundamentalmente por parte de las propias organizaciones de la sociedad civil y de la JEP. Sin embargo, se hace un llamado de apoyo y compromiso a otras instituciones del Estado, atendiendo especialmente a la presencia territorial de la que gozan en el país y a la necesidad de pedagogía para generar los informes en diferentes ciudades y comunidades. En segundo lugar, con respecto los representantes entrevistados, podemos afirmar que las víctimas sí han ganado visibilidad a través de la realización y presentación de los informes, especialmente ante la propia sociedad colombiana y la comunidad internacional. No obstante, se hace evidente la preocupación por el plazo establecido para la presentación de informes -marzo 2020, pero recientemente ampliado- y se hace evidente la preocupación de que esta visibilidad sea efímera. Finalmente, , podemos afirmar que los informes de las organizaciones de víctimas, étnicas y de derechos humanos sí han sido reconocidos como un mecanismo de participación ante la JEP. Sin embargo, se recuerda que la participación de las víctimas en un proceso transicional debe encerrar más elementos que este mecanismo y que este derecho debe ser garantizado a lo largo de todas las etapas del proceso.

Para cerrar este trabajo, nos gustaría resaltar las sensaciones positivas que pueden girar alrededor de la realización y presentación de los informes y que percibimos en las entrevistas realizadas, por ejemplo, la narración de cómo fue la presentación de un informe ante los magistrados y magistradas de la JEP. Esas sensaciones positivas se dejan entrever, no solo porque los informes constituyen la puerta de entrada de las 
víctimas a un proceso transicional decisivo para Colombia, sino porque los informes son un recordatorio de que cada estrategia, cada mecanismo, cada forma y cada etapa de un proceso tiene su propia significación y valor. Como ejemplo de esto, compartimos el siguiente fragmento de una entrevista:

Ellos mismos escribieron el informe, es decir, es la acumulación de sus testimonios. En un círculo estábamos todos y los muchachos se paraban para contar su historia. Luego verlos cuando terminó el acto era increíblemente reparador. Ellos se sentían orgullosos, sentían que estaban aportando, que alguien estaba escuchando su verdad, alguien que para ellos es inalcanzable estaba sentado mirándolos a los ojos, escuchándolos, esto fue increíblemente valioso. No sé cómo es con los otros magistrados, pero lo que tiene que ver con el caso nuestro fue increíblemente reparador. La gente debe asumir que todo el proceso puede ser reparador, no solamente el resultado final. El proceso es reparador para las víctimas por estar acompañándolas en todo momento, por ser muy cautos, por darles toda la información para que no se lleven sorpresas, para que no se sientan utilizadas. (Coalico).

\section{Bibliografía}

Acto Legislativo 01 de 2017, por medio del cual se crea un título de disposiciones transitorias de la Constitución para la terminación del conflicto armado y la construcción de una paz estable y duradera y se dictan otras disposiciones. 4 de abril de 2017. D. O. 50196.

Acuerdo Final de Paz [Gobierno Nacional y FARC-EP]. Acuerdo final para la terminación del conflicto y la construcción de una paz estable y duradera. 12 de noviembre de 2016.

Andreas Forer, Resultados de la participación de las víctimas en la justicia transicional y en la justicia permanente. ¿Cómo se puede hacer más eficiente en escenarios de macrocriminalidad?, en Colombia: un nuevo modelo de justicia transicional, 315-393 (Andreas Forer y Claudia López Díaz, Eds. Deutsche Gesellschaft für Internationale Zusammenarbeit -GIZ-, 2012).

Annette Pearson, Is restorative justice a piece of the Colombian transitional justice puzzle? Restorative Justice, 5 An International Journal, n. ${ }^{\circ}$ 2, 293-308 (2017). https://doi.org/10.1080/20504721.2017.1343419

Bernardo del Rosal Blasco, ¿Hacia el derecho penal de la posmodernidad?, 11 Revista Electrónica de Ciencia Penal y Criminología, n. ${ }^{\circ}$ 8, 1-64 (2009). http://criminet.ugr.es/recpc/11/recpc11-08.pdf

Claudia Medina Aguilar, La Jurisdicción Especial para la Paz (JEP): claridades e incertidumbres de un modelo innovador de justicia transicional, Cuadernos de estrategia, n. ${ }^{\circ}$ 189, 221-232 (2017). https://dialnet.unirioja.es/servlet/ar ticulo? codigo $=6304824$

Comisión Colombiana de Juristas, La participación de las victimas en el Sistema integral de verdad, justicia, reparación y no repetición (2017). http://www.coljuristas.org/documentos/libros_e_informes/Libro_LA_PARTICIPAC I\%C3\%93N_DE_LAS_V\%C3\%8DCTIMAS_CCJ_15_Marzo_2017.pdf

Comisión Colombiana de Juristas, Promover la garantía y exigir los derechos: Guía conceptual sobre el SIVJRNR (2017). http://www.coljuristas.org/documentos/libros_e_informes/la_integralidad_como_garantia_de_no_r epeticion.pdf

Corte Constitucional de Colombia. Sentencia C-084 de 2016 (M. P. Luis Ernesto Vargas Silva; 24 de febrero de 2016).

Corte Constitucional de Colombia. Sentencia C-370 de 2006 (M. Ps. Manuel José Cepeda Espinosa, Jaime Córdoba Triviño, Rodrigo Escobar Gil, Marco Gerardo Monroy Cabra, Alvaro Tafur Galvis, Clara Inés Vargas Hernández, 18 de mayo de 2006).

Corte Constitucional de Colombia. Sentencia C-579 de 2013 (M. P. Jorge Ignacio Pretelt Chaljub 28 de agosto de 2013).

Corte Constitucional de Colombia. Sentencia C-674 de 2017 (M. P. Luis Guillermo Guerrero Pérez; 14 de noviembre de 2017).

Daniel Van Ness \& Karen Strong, Restoring Justice (Editorial Rústica, 2001). 
Enrique Gil Botero, El gobierno nacional y la justicia transicional, en Retos de la implementación de la justicia transicional en Colombia, 17-20 (Sebastián Peñuela Camacho, Eduardo F. Gutiérrez González y María Lucía Zapata Cancelado, Eds., Pontificia Universidad Javeriana, 2018).

Francisco de Roux Rengifo, La Jurisdicción Especial para la paz y la Comisión de la Verdad: retos de la justicia transicional en Colombia, en Retos de la implementación de la justicia transicional en Colombia, 47-69 (Sebastián Peñuela Camacho, Eduardo F. Gutiérrez González y María Lucía Zapata Cancelado, Eds., Pontificia Universidad Javeriana, 2018).

Gabriel Ignacio Gómez Sánchez, Justicia transicional "desde abajo": un marco teórico constructivista critico para el análisis de la experiencia colombiana, 10 Co-Herencia, n. ${ }^{\circ}$ 19, 137-166 (2013). https://doi.org/10.17230/co-herencia. 10.19 .6

Isabella Bueno, Stephan Parmentier \& Elmar Weitekamp, Exploring restorative justice in situations of political violence: the case of Colombia, en Restorative Justice in Transitional Settings (Kerry Clamp, Edit., Routledge, Londres, 2016). https://doi.org/10.4324/9781315723860

Juan Felipe García Arboleda, Los debates de la justicia transicional en Colombia, en Retos de la implementación de la justicia transicional en Colombia, 29-44 (Sebastián Peñuela Camacho, Eduardo F. Gutiérrez González \& María Lucía Zapata Cancelado, Eds., Pontificia Universidad Javeriana, 2018).

Juana Inés Acosta-López \& Cindy Espitia-Murcia, Pasado, presente y futuro de la justicia transicional en el sistema interamericano de derechos humanos, International Law, 15 Revista Colombiana de Derecho Internacional, n. ${ }^{\circ}$ 30, 9-40 (2017). https://doi.org/10.11144/Javeriana.il15-30.ppfj

Julio Andrés Sampedro Arrubla, La re-humanización del sistema penal: una propuesta desde las víctimas y la justicia restaurativa (Pontificia Universidad Javeriana y Grupo Editorial Ibáñez, 2019).

Jurisdicción Especial para la Paz (JEP), Documento guia para la presentación de informes elaborados por organizaciones de victimas, indigenas, negras, afrocolombianas, raizales, palenqueras, Rrom y de derechos humanos colombianas (2018). https://www.jep.gov.co/Sala-de-Prensa/Documents/25.05.18\%208pm\%20SRVR\%20GUIA\%20para \%20la\%20elaboracion\%20y\%20presentacion\%20de\%20informes\%20.pdf

Jurisdicción Especial para la Paz (JEP), Enfoque de género en la JEP. Informe de gestión 2018 (2018). https://www.je p.gov.co/Documents/Transparencia/9.pdf

Jurisdicción Especial para la Paz (JEP), Informe estadístico. Entrega de informes 2019 (2019). https://www.jep.gov.co /Especiales/rendicion/assets/images/informeestadistico.pdf

Ley 1922 de 2018. Por medio de la cual se adoptan unas reglas de procedimiento para la Jurisdicción Especial para la Paz. 18 de julio de 2018. D. O. 50658.

Ley 1957 de 2019. Ley Estatutaria de la Administración de Justicia en la Jurisdicción Especial para la Paz. 6 de junio de 2019. D. O. 50.976.

Lorena Cecilia Vega Dueñas \& Alberto José Olalde Altajeros, La justicia restaurativa como paradigma orientador de la paz: los encuentros restaurativos, ICADE Revista de las Facultades de Derecho, n. ${ }^{\circ} 103,23-35$ (2018). https:// doi.org/10.14422/icade.i103.y2018.001

Louis Bickford, Transitional Justice, en The encyclopedia of genocide and crimes against humanity, 1045-1047 (Dinah Shelton, Ed., Macmillan Publishing, 2004).

Luis Miguel Gutiérrez Ramírez, La obligación internacional de investigar, juzgar y sancionar graves violaciones a los derechos humanos en contextos de justicia transicional, 16 Revista Estudios Socio-Juri\#dicos, n. ${ }^{\circ} 2$ 2, 23-60 (2014). https://doi.org/10.12804/esj16.02.2014.01

Naciones Unidas, Manual sobre programas de justicia restaurativa (2006). https://www.unodc.org/documents/justic e-and-prison-reform/Manual_sobre_programas_de_justicia_restaurativa.pdf

Naciones Unidas \& Universidad Nacional de Colombia, Informe y balance general. Foros nacionales y regionales sobre victimas (2014). http://pensamiento.unal.edu.co/fileadmin/recursos/focos/piensa-paz/docs/foros/informe_b alance_foros_victimas.pdf

Pablo de Greiff, Informe del Relator Especial sobre la promoción de la verdad, la justicia, la reparación y las garantías de no repetición, Pablo de Greiff (2012). Doc. A/HRC/21/46. 
Patricia Linares Prieto, Sobre la Jurisdicción Especial para la Paz, en Retos de la implementación de la justicia transicional en Colombia, 21-25 (Sebastián Peñuela Camacho, Eduardo F. Gutiérrez González \& María Lucía Zapata Cancelado, Eds., Pontificia Universidad Javeriana, 2018).

Reparations, Responsibility \& Victimhood in Transitional Societies, Alternative sanctions before the Special Jurisdiction for Peace: Reflections on International Law and Transitional Justice (2019). https://reparations.qub.ac.uk/asset s/uploads/JEP-Report-Eng.pdf

Tatiana Rincón, Verdad, Justicia \& Reparación, La justicia de la justicia transicional (Editorial Universidad del Rosario, 2010).

Ximena Medellín \& Tatiana Rincón, Digesto de jurisprudencia latinoamericana sobre derechos de las víctimas (Fundación para el Debido Proceso, 2014).

\section{Notas}

[1] Pablo de Greiff, Informe del Relator Especial sobre la promoción de la verdad, la justicia, la reparación y las garantías de no repetición, Pablo de Greiff, 17 (2012). Doc. A/HRC/21/46.

[2] Acuerdo Final de Paz [Gobierno Nacional y FARC-EP]. Acuerdo final para la terminación del conflicto y la construcción de una paz estable y duradera. 12 de noviembre de 2016, 145.

[3] Acuerdo Final de Paz, 145.

[4] Louis Bickford, Transitional Justice, en The encyclopedia of genocide and crimes against humanity, 1045-1047, (Dinah Shelton, Ed., Macmillan Publishing, 2004).

[5] Gabriel Ignacio Gómez Sánchez, Justicia transicional "desde abajo": un marco teórico constructivista crítico para el análisis de la experiencia colombiana, 10 Co-Herencia,.$^{\circ}{ }^{\circ}$ 19, 137-166 (2013).

[6] Tatiana Rincón, Verdad, Justicia \& Reparación, La justicia de la justicia transicional, 26 (Editorial Universidad del Rosario, 2010).

[7] Claudia Medina Aguilar, La Jurisdicción Especial para la Paz (JEP): claridades e incertidumbres de un modelo innovador de justicia transicional, Cuadernos de estrategia, n. ${ }^{\circ}$ 189, 221-232 (2017).

[8] La centralidad de las víctimas en la JEP se ha visto reflejada en las diferentes disposiciones normativas que regulan esta jurisdicción, a saber: Acuerdo Final de Paz, 143; Acto Legislativo 01/2017 (art. transitorio 12); Ley 1957 de 2019 Estatutaria de la JEP (art. 13); y Ley 1922 de 2018 de Procedimiento de la JEP (Título I, Libro I).

[9] En relación al cambio del papel de la víctima en el proceso penal se puede consultar: Bernardo del Rosal Blasco, ¿Hacia el derecho penal de la posmodernidad?, 11 Revista Electrónica de Ciencia Penal y Criminología, n. 8, 1-64 (2009).

[10] Julio Andrés Sampedro Arrubla, La re-humanización del sistema penal: una propuesta desde las víctimas y la justicia restaurativa, 49 (Pontificia Universidad Javeriana y Grupo Editorial Ibáñez, 2019).

[11] Ximena Medellín \& Tatiana Rincón, Digesto de jurisprudencia latinoamericana sobre derechos de las víctimas, 8 (Fundación para el Debido Proceso, 2014).

[12] Acuerdo Final de Paz, 124.

[13] Íd. , 126.

[14] En relación al desarrollo, metodología y hallazgos de los foros de víctimas se puede consultar: Naciones Unidas \& Universidad Nacional de Colombia, Informe y balance general. Foros nacional y regionales sobre victimas (2014).

[15] Acuerdo Final de Paz, 144.

[16] Íd., 128.

[17] Jurisdicción Especial para la Paz (JEP), Enfoque de género en la JEP. Informe de gestión 2018 (2018).

[18] Corte Constitucional de Colombia. Sentencia C-084 de 2016 (M. P. Luis Ernesto Vargas Silva; 24 de febrero de 2016).

[19] Otras sentencias de la Corte Constitucional que se han pronunciado en relación a los derechos de las víctimas en escenarios de justicia transicional son: Sentencia C-370, de 18 de mayo de 2006; Sentencia C-579, de 28 de agosto de 2013; y Sentencia C-674, de 14 de noviembre de 2017.

[20] Luis Miguel Gutiérrez Ramírez, La obligación internacional de investigar, juzgar y sancionar graves violaciones a los derechos humanos en contextos de justicia transicional, 16 Revista Estudios Socio-Juri\#dicos, n. ㅇ 2, $23-60$ (2014).

[21] Al respecto y para conocer un análisis de la jurisprudencia de la Corte IDH desde una perspectiva crítica y propositiva se puede consultar: Juana Inés Acosta-López y Cindy Espitia-Murcia, Pasado, presente y futuro de la justicia transicional en el sistema interamericano de derechos humanos, 15 Revista Colombiana de Derecho Internacional, n. ${ }^{\circ}$ 30, 9-40 (2017).

[22] Acuerdo Final de Paz, 144.

[23] Comisión Colombiana de Juristas, La participación de las victimas en el Sistema integral de verdad, justicia, reparación y no repetición, 13 (2017). 
[24] Patricia Linares Prieto, Sobre la Jurisdicción Especial para la Paz, en Retos de la implementación de la justicia transicional en Colombia, 22 (Sebastián Peñuela Camacho, Eduardo F. Gutiérrez González \& María Lucía Zapata Cancelado, Eds., Pontificia Universidad Javeriana, 2018).

[25] Andreas Forer, Resultados de la participación de las víctimas en la justicia transicional y en la justicia permanente. ¿Cómo se puede hacer más eficiente en escenarios de macrocriminalidad?, en Colombia: un nuevo modelo de justicia transicional, 318 y ss. (Andreas Forer y Claudia López Díaz, Eds. Deutsche Gesellschaft für Internationale Zusammenarbeit -GIZ-, 2012).

[26] Francisco de Roux Rengifo, La Jurisdicción Especial para la paz y la Comisión de la Verdad: retos de la justicia transicional en Colombia, en Retos de la implementación de la justicia transicional en Colombia, 64 (Sebastián Peñuela Camacho, Eduardo F. Gutiérrez González y María Lucía Zapata Cancelado, Eds., Pontificia Universidad Javeriana, 2018).

[27] Reparations, Responsibility \& Victimhood in Transitional Societies, Alternative sanctions before the Special Jurisdiction for Peace: Reflections on International Law and Transitional Justice, 24 (2019).

[28] Julio Andrés Sampedro Arrubla, op. cit., 33.

[29] Patricia Linares Prieto, op. cit., 23.

[30] Andreas Forer, op. cit., 320.

[31] Comisión Colombiana de Juristas, Promover la garantía y exigir los derechos: Guía conceptual sobre el SIVJRNR, 10 (2017).

[32] Andreas Forer, op. cit., 318 y ss.

[33] Comisión Colombiana de Juristas, Promover la garantía y exigir los derechos: Guia conceptual sobre el SIVJRNR, 11 (2017).

[34] Enrique Gil Botero, El gobierno nacional y la justicia transicional, en Retos de la implementación de la justicia transicional en Colombia, 18 (Sebastián Peñuela Camacho, Eduardo F. Gutiérrez González y María Lucía Zapata Cancelado, Eds., Pontificia Universidad Javeriana, 2018).

[35] En relación al alcance de la justicia restaurativa en el Acuerdo de paz se puede consultar: Lorena Cecilia Vega Dueñas \& Alberto José Olalde Altajeros, La justicia restaurativa como paradigma orientador de la paz: los encuentros restaurativos, ICADE Revista de las Facultades de Derecho, n. ${ }^{\circ}$ 103, 23-35 (2018).

[36] Daniel Van Ness \& Karen Strong, Restoring Justice, 14 (Editorial Rústica, 2001).

[37] Annette Pearson, Is restorative justice a piece of the Colombian transitional justice puzzle? 5 An International Journal, $\mathrm{n}$. 2, 293-308 (2017).

[38] En relación a la justicia restaurativa en contextos de justicia transicional también se puede consultar: Isabella Bueno, Stephan Parmentier y Elmar Weitekamp, Exploring restorative justice in situations of political violence: the case of Colombia, en Restorative Justice in Transitional Settings (Kerry Clamp, Edit., Routledge, Londres, 2016).

[39] Juan Felipe García Arboleda, Los debates de la justicia transicional en Colombia, en Retos de la implementación de la justicia transicional en Colombia, 40 (Sebastián Peñuela Camacho, Eduardo F. Gutiérrez González \& María Lucía Zapata Cancelado, Eds., Pontificia Universidad Javeriana, 2018).

[40] La Sala de reconocimiento de verdad entiende por "organización” a toda asociación o agrupación libre de personas que sean víctimas del conflicto armado colombiano o que representen o tengan la finalidad de proteger sus intereses; o tengan en común la promoción, protección y defensa de los derechos humanos. Esto incluye, entre otras, asociaciones espontáneas de víctimas, organizaciones de base, colectivos, plataformas, redes, organizaciones no gubernamentales, etc. Al respecto: Jurisdicción Especial para la Paz (JEP), Documento guía para la presentación de informes elaborados por organizaciones de víctimas, indígenas, negras, afrocolombianas, raizales, palenqueras, Rrom y de derechos humanos colombianas (2018).

[41] Este mecanismo de participación también fue recogido por el Acuerdo Final de Paz, 154; y por el Acto Legislativo $01 / 2017$ (art. transitorio 15).

[42] Jurisdicción Especial para la Paz (JEP), Documento guía para la presentación de informes elaborados por organizaciones de víctimas, indígenas, negras, afrocolombianas, raizales, palenqueras, Rrom y de derechos humanos colombianas (2018).

[43] Jurisdicción Especial para la Paz (JEP), Documento gui\#a para la presentación de informes elaborados por organizaciones de vi\#ctimas, indígenas, negras, afrocolombianas, raizales, palenqueras, Rrom y de derechos humanos colombianas, (2018).

[44] Íd.

[45] A manera de ejemplo, en agosto de 2018, uno de los actos públicos y simbólicos que se realizó fue la entrega y recepción del informe a la JEP por parte de la Organización Nacional Indígena de Colombia (ONIC). Al respecto se puede consultar: https://www.jep.gov.co/Sala-de-Prensa/Paginas/Onic-hace-entrega-de-informes-a-la-JEP.aspx

[46] A manera de ejemplo, en diciembre de 2018, la Sala de Reconocimiento de verdad recibió en Apartadó (Antioquia) la fase oral del informe mixto sobre los hechos de violencia sexual y violencia basada en género ocurridos en el marco del conflicto armado, en el Urabá antioqueño y chocoano. Al respecto se puede 
consultar: https://www.jep.gov.co/Sala-de-Prensa/Paginas/Un-acto-de-dignificaci\%C3\%B3n-de-las-v\%C3\%ADctim as-de--violencia-sexual-y-violencia-de-g\%C3\%A9nero-en-Urab\%C3\%A1.aspx

[47] El Departamento de Gestión Territorial trabajó hasta noviembre del 2018 con veinte (20) enlaces territoriales. Al respecto: Jurisdicción Especial para la Paz (JEP), Informe estadistico. Entrega de informes 2019 (2019).

[48] Jurisdicción Especial para la Paz (JEP), Documento gui\#a para la presentación de informes elaborados por organizaciones de vi\#ctimas, indígenas, negras, afrocolombianas, raizales, palenqueras, Rrom y de derechos humanos colombianas (2018).

[49] En este sentido también se pronunció el Acuerdo Final de Paz, 155.

[50] Jurisdicción Especial para la Paz (JEP), Documento gui\#a para la presentación de informes elaborados por organizaciones de vi\#ctimas, indígenas, negras, afrocolombianas, raizales, palenqueras, Rrom y de derechos humanos colombianas, (2018).

[51] Íd.

[52] Acuerdo Final de Paz, 144. Cursivas propias.

[53] Naciones Unidas, Manual sobre programas de justicia restaurativa (2006).

[54] Íd.

[55] De los siete (7) representantes elegidos por las propias organizaciones para ser las personas entrevistadas, dos (2) de ellas se identificaron como víctimas y cinco (5) son abogados de las organizaciones.

[56] Las organizaciones que no contestaron la invitación a realizar la entrevista o que contestaron manifestando interés en la investigación, pero que finalmente no delegaron a un representante o que fue imposible la disponibilidad por cuestiones de agenda, fueron las siguientes: Asociación Nacional de Afrocolombianos Desplazados (AFRODES); BENPOSTA Nación de muchachos Colombia; Víctimas del atentado al Club El Nogal; Colectivo sociojurídico Orlando Fals Borda; Comisión intereclesial de justicia y paz; Fundación comité de solidaridad con los presos políticos; Corporación jurídica Yida Castro; Corporación mujer sigue mis pasos; REINICIAR Corporación para la defensa y promoción de los derechos humanos; Corporación social para la asesoría y capacitación comunitaria; Humanidad vigente Corporación jurídica; Fundación para la protección de los derechos de las víctimas; Organización mil víctimas del conflicto; Red de mujeres víctimas y profesionales; Red nacional de mujeres; y Corporación Sisma mujer.

[57] En este sentido, vale la pena destacar que la Procuraduría General de la Nación y la JEP han venido creado estrategias de cooperación, con el fin de fortalecer la presencia territorial y el papel de las víctimas en el SIVJRNR. Al respecto se puede consultar: https://www.jep.gov.co/Sala-de-Prensa/SiteAssets/Paginas/Comunicado-034-de-2019---La-JEP-y-la-Proc uradur\%C3\%ADa-lideran-estrategia-para-fortalecer-la-participaci\%C3\%B3n-de-las-v\%C3\%ADctimas/34.\%20Com unicado\%2034\%20-\%20JEP\%20y\%20Procuraduria.pdf

[58] $\mathrm{Al}$ respecto es importante destacar que, en caso de que algunas organizaciones cuenten con información relevante para los casos de competencia de la JEP, pero no cuenten con herramientas para procesarla como informes, podrán presentar ante la Secretaría Ejecutiva una solicitud de acompañamiento especial con el fin de orientar la elaboración y presentación del informe. Jurisdicción Especial para la Paz (JEP), Documento gui\#a para la presentación de informes elaborados por organizaciones de vi\#ctimas, indígenas, negras, afrocolombianas, raizales, palenqueras, Rrom y de derechos humanos colombianas (2018).

[59] Al respecto se puede consultar: https://www.jep.gov.co/Sala-de-Prensa/Paginas/La-JEP-fortalece-su-presencia-territor ial-con-una-oficina-en-Corozal-Sucre.aspx https://www.jep.gov.co/Sala-de-Prensa/Paginas/LA-JEP-abre-en-C\%C3\% BAcuta-su-segunda-sede-territorial.aspx https://www.jep.gov.co/Sala-de-Prensa/Paginas/La-JEP-abre-en-Pasto-una-n ueva-sede-territorial.aspx

* Artículo de investigación. Este artículo hace parte del proyecto de investigación "Ley de procedimiento de la Jurisdicción Especial para la Paz: aciertos, debilidades y desafíos del derecho penal en contextos de justicia transicional”, financiado por el Instituto Colombo Alemán para la Paz-CAPAZ-. La autora agradece a cada una de las personas entrevistadas por su disponibilidad y aporte fundamental a este trabajo. Así mismo, agradece a Carlos Vergara, Andrés Puentes, Ángela Gómez, Juanita Sarquis, Mateo Merchán, Santiago Guerrero, Sara Astrálaga y Valentina Neira, estudiantes de Derecho de la Pontificia Universidad Javeriana, quienes acompañaron a la autora en algunas entrevistas y colaboraron en la sistematización de la información obtenida en las mismas.

\section{Licencia Creative Commons CC BY 4.0}

Cómo citar este artículo: Lorena Cecilia Vega Dueñas, Participación de las victimas en la JEP: especial referencia a los informes de las organizaciones de victimas, étnicas y de derechos humanos, 69 Vniversitas (2020). https:/ /doi.org/10.11144/Javeriana.vj69.pvje 